Monleón Oliva, Vicente.

Alumno de 2o curso del Programa de Doctorado en Didácticas Específicas (Artes Visuales), Universidad de Valencia, Departamento de Didáctica de la Expresión Musical, Plástica y Corporal, grupo de investigación en pedagogías culturales CREARI.

\title{
Arte y educación en diversidad sexual. La percepción de la homosexualidad en una clase de 60 curso a través de la técnica del dibujo
}

\section{Arts and education in sexual diversity. The perception of homosexuality in 6th course through drawing technique}

TIPO DE TRABAJO: Comunicación.

PALABRAS CLAVE

Diversidad sexual, homosexualidad, educación y dibujo.

KEY WORDS

Sexual Diversity, homosexuality, education and drawing.

RESUMEN

La sexualidad es uno de los temas tabú de los que adolece la educación en el territorio occidental. Se manifiesta una latente reticencia hacia su tratamiento en las aulas, más aún cuando se apuesta por abordarla desde la diversidad. Pero precisamente para avanzar es necesario apostar por esta pluralidad, romper con la norma establecida y desmontar el patriarcado que construye una única identidad entre el colectivo infantil que aparece diferenciado según su sexo. Apostando por superar los convencionalismos de la temática cuando esta se relaciona con el uso del preservativo o la menstruación. No obstante, con la iniciativa propuesta "Arte y educación en diversidad sexual" se pretende posicionar al grupo de estudiantes de sexto curso de una escuela pública de inclusión de Valencia desde un posicionamiento que permita entender la diversidad y respetarla, incluso normativizarla. Este conjunto tiene que contestar a una cuestión a través de la expresión plástica: "Dibuja a dos personas del mismo sexo mostrando su amor en público, ¿cómo te las imaginas?". El ejercicio permite conocer cuáles son los conocimientos de un grupo mixto formado por 18 estudiantes. Averiguando como es la percepción de la clase hacia quienes forman parte del colectivo LGBT. Iniciativas como la presentada son necesarias para que el conjunto de discentes de las escuelas sea educado de una manera global, integral y conducido hacia valores de tolerancia y respeto. La educación debe de apostar por una diversidad sexual que prevenga los comportamientos homofóbicos que se desarrollan a medida que quien estudia crece, como consecuencia de no haber recibido una educación que contemple posibilidades más allá de la heteronormatividad.

\section{ABSTRACT}

Sexuality is one of the taboo topics that education suffers in the western territory. There is a latent reticence towards its treatment in the classrooms, even more when it is committed to address it from diversity. But precisely to advance it is necessary to bet on this plurality, to break with the established norm and to dismantle the patriarchy that builds a unique identity among the infantile group that appears differentiated according to its sex. Betting on overcoming the conventions of the theme when it is related to the use of condoms or menstruation. However, the proposed initiative "Art and education in sexual diversity" aims to position the group of sixthyear students of a public school of inclusion in Valencia from a position that allows to understand diversity and respect it, even standardize it. This group has to answer an issue through the plastic expression: "Draw two people of the same sex showing their love in public, how do you imagine them?". The exercise allows knowing what the knowledge of a mixed group of 18 students is. Finding out how is the perception of the class towards those who are part of the LGBT collective. Initiatives such as the one presented are necessary so that all of the students in the schools are educated in a global, integral and conducted way towards values of tolerance 
and respect. Education should bet on a sexual diversity that prevents homophobic behaviors that develop as the student grows, as a result of not having received an education that contemplates possibilities beyond heteronormativity.

\section{INTRODUCCIÓN}

El concepto de homosexualidad es un tópico debatido, cuestionado y criticado (Dowson, 1998) a lo largo de la historia (Boswell, 1980 y 1994; Spencer, 1995; Woods, 1998), pero en líneas generales "se entiende por persona homosexual la atracción afectiva o sexual hacia las personas del mismo género o sexo" (Soler-Quílez, 2015, p. 2954).

Hay una corriente orientada a contribuir a la idea del innatismo biológico como explicación de la homosexualidad, es decir, "se es como se nace" (Escobar, 2007:85). Todo esto se debe, según autores como Tancredi (2005) a que el cerebro durante su gestación y crecimiento no recibe los niveles de testosterona necesarios (de manera que el niño es gay) o los recibe en cantidades superiores a las necesarias (la niña es lesbiana).

En las antiguas civilizaciones como la romana, la maya, la sumeria, la china de la dinastía y/o la griega; las prácticas sexuales entre personas del mismo sexo no solo están permitidas, sino que incluso llegan a considerarse sagradas (Dowson, 1998; Matthews, 1994). Ya en época de Platón, en la Grecia Clásica, se alude a un mito que explica la homosexualidad en hombres y mujeres (Escobar, 2007). Según la mitología clásica los seres andróginos se presentan como bisexuales, es decir, están formados por un hombre y una mujer fusionados en un único cuerpo. Hasta el momento en que Zeus decide castigarles y los subdivide en dos mitades quienes están condenadas a buscarse durante toda la vida, ya que cada individuo solo tiene medio corazón. Esto explica, por un lado, las relaciones heterosexuales y el amor romántico. Pero, por otro lado:

Sin embargo, según el mismo Platón, la atracción de los hombres por otros hombres y de las mujeres por otras mujeres se debía a que hombres y mujeres primitivos también eran dobles, estaban constituidos por dos mujeres o dos hombres unidos. Si descienden de una rama hombre de la humanidad primitiva, los hombres buscan otro hombre y si descienden de la rama mujer, las mujeres se sienten atraídas por las mujeres. (Escobar, 2007, p. 81)

No obstante, con la llegada del cristianismo y de la moral que dicha religión conlleva, en la Edad Media la homosexualidad es considerada un delito perseguido y castigado (Flandrin, 1987; Helminiak, 2003). "Es claro que para la Iglesia católica los actos homosexuales no pueden aprobarse en ningún caso" (Collignon, 2011, p. 141). Entre algunos de los rastreadores de estas prácticas consideradas como obscenas y lascivas destacan los teólogos Tomás de Aquino y San Agustín.

Con todo, se inculca entre la sociedad un pensamiento hermenéutico en lo que se relaciona con las prácticas sexuales (Escobar, 2007); no solo la homosexualidad pasa a ser condenada sino también la masturbación, el sexo oral y cualquier otra práctica que la iglesia pueda considerar como un atentado contra la naturaleza humana. Ciertamente, con arraigo en esta tradición, a día de hoy sentimientos de repudia y odio hacia los homosexuales continúan vigentes. Que, en consecuencia hay que combatir y exterminar.

Esta tradición histórico-cultural conlleva a sentimientos de odio, rechazo, desaprobación y violencia hacia quienes son diferentes, diversos, hacia quienes no cumplen unos patrones arraigados en la sociedad heteropatriarcal. Destacándose como problemática más significativa la escasez de importancia que se le ofrece a introducirla en las aulas. Hay una reticencia e incluso miedo a ser juzgados por las familias por intentar normativizar aquello que un grupo trata de invisibilizar. 


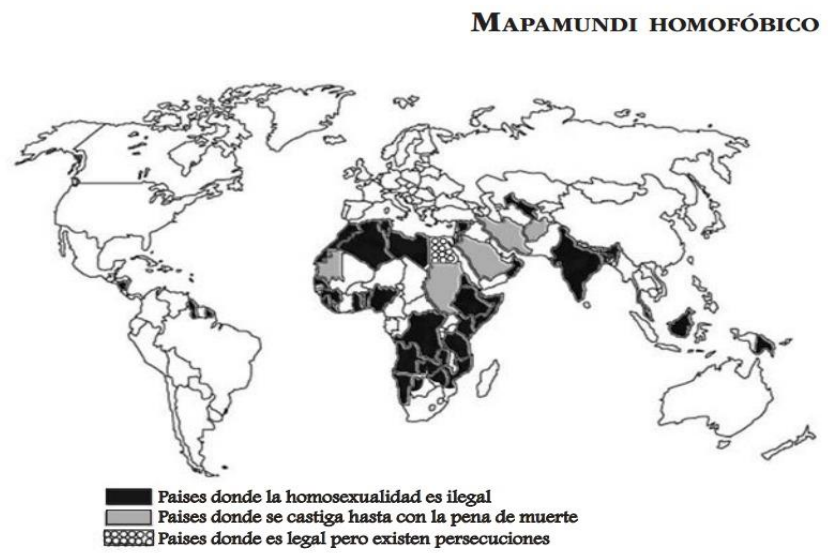

Figura 1. Mapamundi Homofóbico

Partiendo de esta situación fehaciente al contexto escolar occidental actual se considera oportuno acercar el concepto de homosexualidad al último curso de la Educación Primaria, ya que es el periodo educativo que corresponde con la pubertad e inicio del desarrollo sexual de las personas. Se pretende conocer qué ideas tiene un grupo compuesto por 18 estudiantes ( 8 niños y 8 niñas) acerca de las personas homosexuales a través de la expresión plástica que sigue la técnica del dibujo. El colectivo tiene que plasmar sobre una hoja en blanco de manera individual cómo se imagina a dos personas del mismo sexo manifestando su amor en público. Asimismo, también se propone como objetivos la visibilización de colectivos minoritarios como es el LGBT en lo referentes a gays y lesbianas; así como el desmantelamiento de posibles estereotipos manifestados entre el grupo de discentes en lo que se relaciona con las personas no-normativas.

Dos hipótesis de partida que quieren comprobarse con este estudio de caso son: por un lado, la posibilidad de una mayor visibilización del grupo de hombres gays que de mujeres lesbianas; por otro lado, la existencia de una creencia generalizada que destina cruces entre el sexo, la sexualidad y la orientación sexual, un pensamiento heteronormativo que define a las mujeres lesbianas con sexo de mujer y género masculino, mientras que se percibe a los homosexuales con sexo de hombre y género femenino.

\section{METODOLOGÍA}

Se recurre a una metodología de investigación de tipo mixto (Sánchez, 2014; Cedeño, 2012), ya que combina peculiaridades de la cuantitativa y la cualitativa. De tal marena que previamente se procede a un análisis cualitativo de la información que el grupo de discentes comparte a través de sus creaciones artísticas. Pero dicha información se cuantifica y se comparte visualmente en forma de gráficas de barras, recurriéndose por tanto a aspectos de la investigación de corte cuantitativo.

Según Hernández, Fernández y Baptista (2003) los Ilamados multi-métodos, métodos mixtos o triangulación metodológica:

[‥] Representan el más alto grado de integración o combinación entre los enfoques cualitativo y cuantitativo. Ambos se entremezclan o combinan en todo el proceso de investigación, o, al menos, en la mayoría de sus etapas (...) agrega complejidad al diseño de estudio; pero contempla todas las ventajas de cada uno de los enfoques. (p. 21)

También se destaca la corriente del a/r/tography (Irween, Beer, Springgay, Grauer, Xiong y Bickel, 2006; la Jevic y Springgay, 2008; Siegesmund, 2012) que empapa esta investigación. Según esta tendencia metodológica la persona que enseña y aquella que investiga se fusionan en un único individuo que pretende indagar sobre la realidad en la que se encuentra a través de cualquier rama de las artes. 


\section{DESARROLLO}

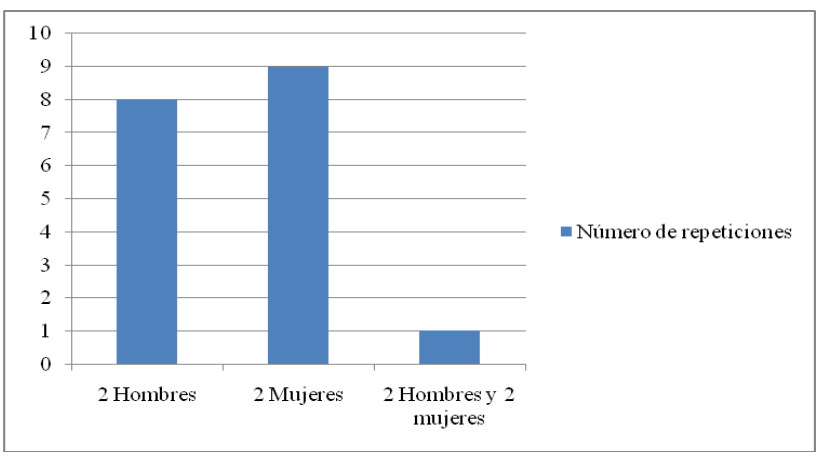

Figura 2. Tipo de pareja homosexual dibujada por los niños y niñas de la clase de sexto

En esta gráfica se aprecia que 9 niñas dibujan una pareja de mujeres lesbianas y 8 chicos a dos hombres homosexuales. Quedando solo 1 niño que decide recrear tanto una pareja gay como otra lésbica. Hay una tendencia a que chicos dibujen hombres y chicas a mujeres. Esto sorprende ya que se constata que la homosexualidad lésbica está bastante visibilizada en la clase, al menos por parte de las mujeres. Este hallazgo sorprende ya que se constata que la homosexualidad lésbica está bastante visibilizada en la clase, al menos por parte de las mujeres (Cencerrado-Malmierca y Cedeira-Serantes, 2006; Mujika, 2007). El propio grupo argumenta que cuando se es chica resulta más sencillo dibujar a personas de su mismo sexo; igual que ocurre con el grupo de niños.

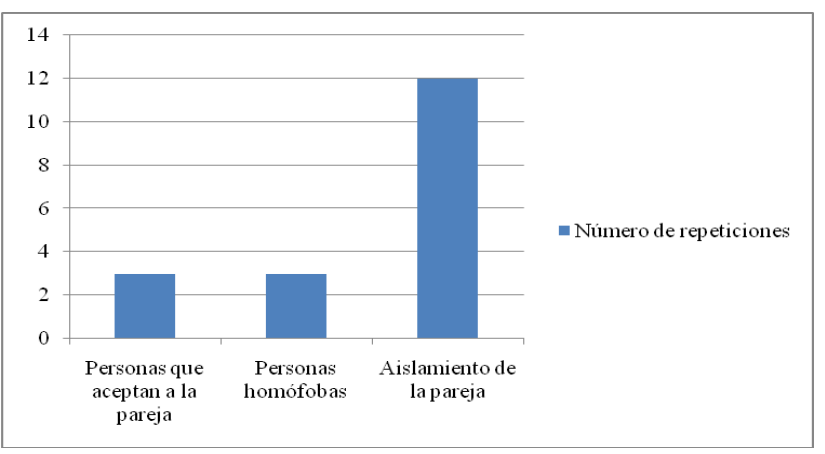

Figura 3. Ambiente que envuelve a la pareja homosexual dibujado por los niños y niñas de clase de sexto

Respecto a esta categoría se aprecia que la mayoría de educandos, 12 concretamente, perciben que las parejas homosexuales están aisladas de la realidad o que, aunque estén en un lugar público se muestran en solitario. Este es un estereotipo con necesidad de romper, la tendencia a aislar a las personas del colectivo LGBT. Se aprecia que 3 discentes dibujan a la pareja homosexual rodeada de personas que las aceptan y otros 3 en un ambiente homofóbico. Por ello, se advierte la necesidad de trabajar para que sean conscientes de la importancia que que reside en la eliminación de la homofobia en la sociedad occidetal actual.

Los hombres en su mayoría (Soler-Quílez, 2015; Sánchez, 2009; Dowson, 1998; Mercado, 2009; Tin, 2008) odian a sus respectivos homosexuales, sobre todo a los que son penetrados; para ellos son como mujeres y los consideran inferiores. Sánchez (2009) lo explica de esta manera:

La homofobia es el miedo o rechazo hacia la homosexualidad. Se aplica sólo a los hombres, pero no a las lesbianas; o más precisamente sólo a los hombres que son penetrados analmente en la relación sexual; o sólo a los que se visten de mujer. Al hombre que penetra a otro en muchos casos ni siquiera se le considera homosexual y, por tanto no es sujeto de homofobia. No ocurre lo mismo con el hombre penetrado, al cual se desprecia por considerarlo afeminado. (p. 116)

La homofobia es un resorte del machismo perdurado a lo largo de los siglos. Así se manifiesta con insultos dirigidos a la agresión de los miembros del colectivo LGBT (lesbianas, gays, bisexuales y transexuales). Por ejemplo, esto ocurre con el vocablo "maricón" (Peixoto, Fonseca, Almeida y Almeida, 2012). Cuando los hombres lo utilizan para insultarse entre ellos, pese a ser heterosexuales, están 
reivindicando su superioridad respecto a las mujeres, según prejuicios sociales se cree que estos no lloran, todos son fuertes, rudos, toscos, etc. "El temor a que predomine la parte femenina que llevan los hombres heterosexuales desencadenan [ $\cdots]$ sentimientos en contra de quienes no coinciden con sus estereotipos y son [‥] el origen de la homofobia" (Escobar, 2007, p. 84). Asimismo, la homofobia radica en exclusión social (Escobar, 2007; Lamas, 2004). Los principios e ideología de una sociedad del patriarcado y con autoritarismo causan un fuerte rechazo hacia las personas homosexuales.

A continuación se muestran los ejemplos más significativos:

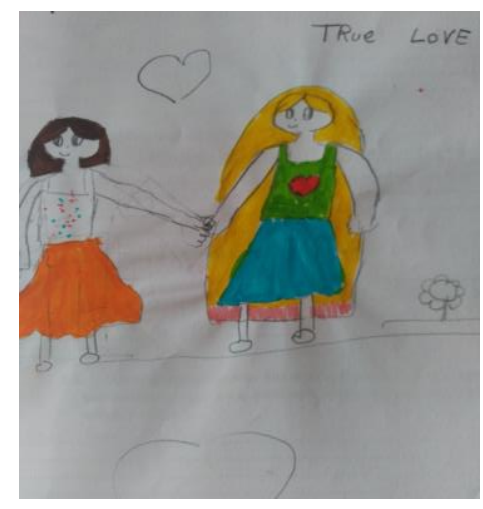

Figura 4. Pareja de mujeres

Este dibujo está hecho por una niña de la clase. Sirve para mostrar el ejemplo de que una gran mayoría de la clase asocia la idea de homosexualidad con la de relación amorosa. En el dibujo se explicita directamente con la frase "true love". Sirve para romper con los estereotipos homosexuales. En este caso, ambas mujeres visten faldas y una de estas tiene un pelo desmesuradamente largo.

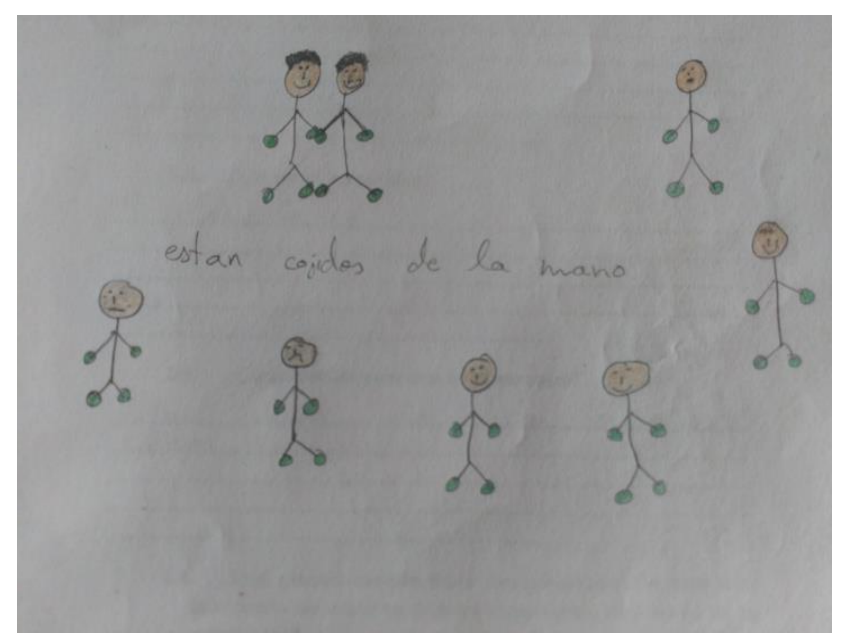

Figura 5. Pareja de hombres rodeada de otros varones [Figura].

Este segundo ejemplo ofrecido por un niño del aula muestra como las parejas homosexuales (en este caso de hombres) se encuentran inmersas en la sociedad. En esta se encuentran personas que apoyan sus relaciones, otros que les atacan y miembros que se muestran indiferentes. Resulta significativo por el amplio rango de posibilidades y actitudes sociales que muestra. 


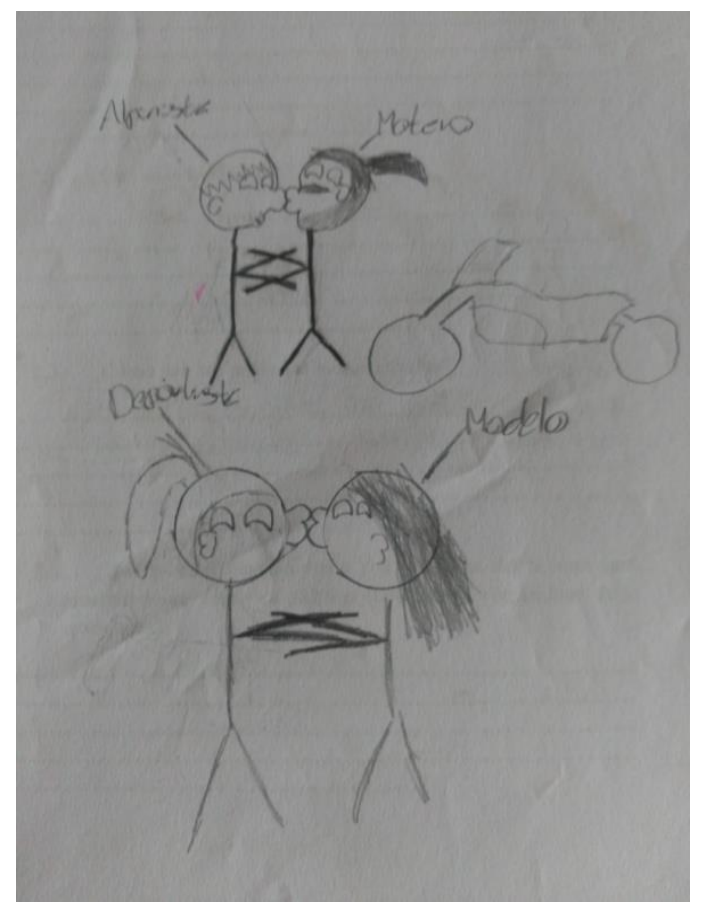

Figura 6. Dos parejas homosexuales [Figura].

Finalmente, el último dibujo también creado por un niño muestra tanto una pareja gay como otra lésbica. De esta manera da a conocer ambas posibilidades de relación homosexual. También, se vuelve significativo el ejemplo ya que muestra un gran número de posibilidades que rompen con los estereotipos: alpinista y motero; deportista y modelo.

En relación con todas las aportaciones destaca que todos los dibujos son de personas más mayores que las del grupo de la clase. No asimilan que la homosexualidad es un tipo de sexualidad que se presenta desde las primeras edades de vida. Por ello hay que educarlos abiertamente y utilizar la propuesta de intervención como un modelo para entender que en un momento dado compañeros y compañeras de clase tienen la posibilidad de compartir abiertamente su orientación sexual. Rompiendo con el miedo que surge hacia dicho tema. Por ejemplo una niña comenta: "pero, ¿estas pruebas que nos vas a pasar es para saber si somos homosexuales?".

\section{CONCLUSIONES}

Con el trabajo compartido se cumple con los objetivos planteados previamente, así como también se responde a las dos hipótesis de partida. Ayudando a construir conocimiento en lo que respecta a temas de diversidad sexual. Para seguir mejorando en educación y conseguir procesos de enseñanza-aprendizaje óptimos que se encaminen hacia un aprendizaje integral de quienes son discentes.

Por un lado, tras la revisión bibliográfica y a raíz de los comentarios compartidos por el grupo de estudio se verifica la reticencia que hay en la implementación de actividades sobre diversidad sexual en las aulas, incomodidad manifestada tanto en las familias como en las plantillas docentes; desencadenando sentimientos de negación hacia dicha realidad por parte de quienes estudian. A posteriori generando homofobia en la sociedad. Por ello, si se desea prevenir la aparición de dicho sentimiento de odio e incomprensión de quien no cumple con la normatividad, es necesario hablar abiertamente del tema, normativizándolo y visibilizándolo.

Por otro lado, sorprende la visibilidad que tiene el colectivo de mujeres lesbianas entre quienes configuran la clase, sobre todo entre quienes son niñas. Por ello se aprecia que una tarea idónea para con el colectivo infantil es trabajar y construir empatía hacia dichos grupos. La visibilidad es el primer paso, partir de este momento se debe potenciar en las aulas de Primaria una educación sexual de calidad que apueste por la prevención de la homofobia, no por combatirla una vez ya está desarrollada entre un grupo de menores.

Finalmente, se manifiesta la creencia generaliza de determinados estereotipos fehacientes a la persona homosexual. Los más significativos en este grupo son: el prejuicio de aislamiento del colectivo y la atribución de ciertas feminidades hacia el hombre gay y masculinidades destinadas a la mujer lesbiana. 
A partir de estas conclusiones se plantean como perspectiva de futuro la necesidad y el reto de ampliar la muestra de estudio tanto en número como en condiciones contextuales de la población seleccionada. Planteando esta misma actividad para grupos estudiantiles de diferentes escuelas: públicas, privadas y concertadas. Anhelando el triunfo de la llegada de estos temas a la enseñanza religiosa, que necesita abrir sus puertas hacia realidades latentes como lo es la diversidad sexual, siempre en base a la tolerancia y el respeto.

\section{FUENTES REFERENCIALES}

Boswell, J. (1980). Christianity, social intolerance, and homosexuality. Chicago: Universidad de Chicago Press.

Boswell, J. (1994). Same-sex unions in premodern Europe. Nueva York: Villerd Books.

Cedeño, N. (2012). La investigación mixta, estrategia andragógica fundamental para fortalecer las capacidades intelectuales superiores. Revista Res Non Verba, 2(2), 17-36.

Cencerrado-Malmierca, L.M. y Cedeira-Serantes, L. (2006). La visibilidad de lesbianas y gais en la literatura infantil y juvenil editada en España. Educación y Biblioteca, 152, 89-102.

Collignon, M.M. (2011). Discursos sociales sobre la sexualidad: narrativas sobre diversidad sexual y prácticas de resistencia. Nueva época, 16, 133-160.

Dowson, T.A. (1998). Homosexualitat, teoría queer i arqueologia. Cota Zero, 14, 81-87.

Escobar, J. (2007). Diversidad sexual y exclusión. Revista Colombiana de Bioética, 2(2), 77-94.

Flandrin, J.L. (1987). La vida sexual matrimonial en la sociedad antigua: de la doctrina de la iglesia a la realidad de los comportamientos. En A. Ariès, M. Bejín, M. Focault, et al., Sexualidades occidentales. México: Paidós.

Irwin, R.L., Beer, R., Springgay, S., Grauer, K., Xiong, G. y Bickel, B. (2006). The rhizomatic relations of a/r/tography. Studies in Art Education, 48(1), 70-88.

Helminiak, D.A. (2003). Lo que la Biblia realmente dice sobre la homosexualidad. Barcelona: Egales.

Hernández, R., Fernández, C. y Baptista, P. (2003). Metodología de la investigación (3a ed.). México: Editorial Mc Graw-Hill, pp.21.

La Jevic, L. y Springgay, S. (2008). A/r/tography as an ethics of embodiment: Visual journals in preservice education. Qualitative inquiry, 14(1), 67-89.

Lamas, M. (2004). Explicar la homofobia. A primer plana. Revista de género y comunicación, 3 [archivo en línea]. Recuperado de: www.aprimeraplana.org/www/No.11/paginas/homofobia.htm [Consulta: 2018, 7 de abril].

Matthews, K. (1994). Anarchacology of homosexuality? Perspectivesf rom de Classical World. En A.S. Cottam, D. Dungworth, S. Scott y J. Taylor (eds.), Theoretical Roman Arcacology Conference 94. Oxford: Oxbow Books.

Mercado, J. (2009). Intolerancia a la diversidad sexual y crímenes por homofobia. Un análisis sociológico. Sociológica (México), 24(69), 123-156.

Mujika, I. (2007). Visibilidad y participación social de las mujeres lesbianas en Euskadi. Euskadi: Ararteko.

Peixoto, J.M., Fonseca, L., Almeida, A. y Almeida, L. (2012). Escuela y diversidad sexual - ¿Qué realidad? Educaçao em Revista. Belo Horizonte, 28(3), 143-158.

Sánchez, A.R. (2009). Cuerpo y sexualidad, un derecho: avatares para su construcción en la diversidad sexual. Sociológica, 69, 101-122.

Sánchez, M.C. (2014). La dicotomía cualitativo-cuantitativo: posibilidades de integración y diseños mixtos. Campo Abierto, vol. monográfico, 11-30. 
Siegesmund, R. (2012). Dewey through a/r/tography. Visual Arts Research, 38(2), 99-109.

Soler-Quílez, G. (2015). La diversidad afectivo-sexual en el cómic: material didáctico para el ámbito educativo. En M.T. Tortosa, J.D. Álvarez y N. Pellín (coords.), XIII Jornadas de Redes de Investigación en Docencia Universitaria. Nuevas estrategias organizativas y metodologías en la formación universitaria para responder a la necesidad de adaptación y cambio. Universidad de Alicante.

Spencer, C. (1995). Homosexuality: a history. Londres: Fourth Estate.

Tancredi, L. (2005). Hard wired Behavior. What neuroscience reveals about morality. Nueva York: Cambridge.

Tin, L.G. (2008). El rostro múltiple de la homofobia. Cuba: Centro Nacional de Educación Sexual. Proyecto sobre Diversidad Sexual.

Woods, G. (1998). A history of gay literatura: themale tradition. New Haven: Yale University Press. 